PROCEEDINGS OF THE

AMERICAN MATHEMATICAL SOCIETY

Volume 139, Number 4, April 2011, Pages 1291-1298

S 0002-9939(2010)10562-2

Article electronically published on September 2, 2010

\title{
ON THE RESTRICTION OF THE HERMITIAN EISENSTEIN SERIES AND ITS APPLICATIONS
}

\author{
SHOYU NAGAOKA AND YOSHITUGU NAKAMURA \\ (Communicated by Wen-Ching Winnie Li)
}

\begin{abstract}
We introduce a simple construction of a Siegel cusp form obtained by taking the difference between the Siegel Eisenstein series and the restricted Hermitian Eisenstein series. In addition, we present applications of the Siegel cusp form.
\end{abstract}

\section{INTRODUCTION}

In the present paper, we introduce a simple construction of a Siegel cusp form of degree 2. The Siegel cusp form is realized as the difference between the Siegel Eisenstein series and the restriction of the Hermitian Eisenstein series to the Siegel half-space.

The proposed construction has an advantage because the Fourier coefficient is explicitly computable and has a number of applications. As the first application, we introduce a new description of Cohen's function (Theorem 5.2). Second, explicit formulas for the Fourier coefficients of Igusa's cusp form of weights 10 and 12 are presented (Corollary [5.5). Finally, we refer to the $p$-adic Siegel cusp forms.

\section{Hermitian and Siegel modular forms}

We start by recalling the definition and basic characteristics of Hermitian and Siegel modular forms.

The Hermitian half-space of degree 2 is defined as

$$
\mathbb{H}_{2}:=\left\{Z \in M_{2}(\mathbb{C}) \mid \frac{1}{2 i}\left(Z-{ }^{t} \bar{Z}\right)>0\right\}
$$

and contains the Siegel half-space of degree 2,

$$
\mathbb{S}_{2}:=\left\{Z \in \mathbb{H}_{2} \mid Z={ }^{t} Z\right\}
$$

as a submanifold. Let $\mathbb{K}$ be an imaginary quadratic number field with discriminant $d_{\mathbb{K}}$ and ring of integers $\mathcal{O}=\mathcal{O}_{\mathbb{K}}$. Then, the Hermitian modular group of degree 2 over $\mathbb{K}$ is defined as

$$
\Gamma_{2}(\mathcal{O}):=\left\{M \in M_{4}(\mathcal{O}) \mid{ }^{t} \bar{M} J_{2} M=J_{2}\right\}, \quad J_{2}=\left(\begin{array}{cc}
0 & -1_{2} \\
1_{2} & 0
\end{array}\right) .
$$

Received by the editors December 22, 2009 and, in revised form, April 16, 2010 and April 28, 2010

2010 Mathematics Subject Classification. Primary 11F55; Secondary 11F46.

Key words and phrases. Siegel modular forms, Hermitian modular forms.

(C)2010 American Mathematical Society Reverts to public domain 28 years from publication 
The group $\Gamma_{2}(\mathcal{O})$ acts on $\mathbb{H}_{2}$ by fractional linear transformation $Z \mapsto M\langle Z\rangle:=$ $(A Z+B)(C Z+D)^{-1}, M=\left(\begin{array}{l}A B \\ C D\end{array}\right) \in \Gamma_{2}(\mathcal{O})$. If $\nu$ is an abelian character of $\Gamma_{2}(\mathcal{O})$, then the space $M_{k}\left(\Gamma_{2}(\mathcal{O}), \nu\right)$ of Hermitian modular forms of weight $k$ and character $\nu$ with respect to $\Gamma_{2}(\mathcal{O})$ consists of all holomorphic functions $F: \mathbb{H}_{2} \longrightarrow \mathbb{C}$ satisfying

$$
F(M\langle Z\rangle)=\nu(M) \operatorname{det}(C Z+D)^{k} F(Z)
$$

for all $M=\left(\begin{array}{ll}A & B \\ C & D\end{array}\right) \in \Gamma_{2}(\mathcal{O})$. We denote by $M_{k}\left(\Gamma_{2}(\mathcal{O}), \nu\right)^{s y m}$ the subspace consisting of symmetric Hermitian modular forms characterized by

$$
F\left({ }^{t} Z\right)=F(Z)
$$

A typical example of a symmetric Hermitian modular form is given by the Hermitian Eisenstein series

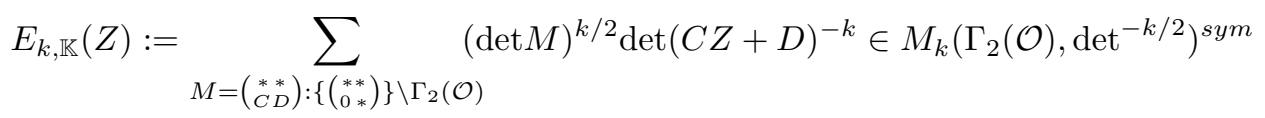

for even $k>4$. Moreover, $E_{4, \mathbb{K}} \in M_{4}\left(\Gamma_{2}(\mathcal{O}) \text {, } \operatorname{det}^{-2}\right)^{\text {sym }}$ is constructed as the Maass lift (cf. Krieg [9]).

Each $F \in M_{k}\left(\Gamma_{2}(\mathcal{O})\right.$, $\left.\operatorname{det}^{l}\right)$ possesses a Fourier expansion of the form

$$
F(Z)=\sum_{0 \leq H \in \Lambda_{2}(\mathbb{K})} A(H ; F) \exp [2 \pi i \operatorname{tr}(H Z)],
$$

where $\Lambda_{2}(\mathbb{K})$ is a lattice in $\mathrm{Her}_{2}(\mathbb{C})$ defined by

$$
\Lambda_{2}(\mathbb{K}):=\left\{\left(\begin{array}{ll}
a & t \\
\bar{t} & b
\end{array}\right) \in M_{2}(\mathbb{K}) \mid a, b \in \mathbb{Z}, \sqrt{d_{\mathbb{K}}} t \in \mathcal{O}\right\} .
$$

The Siegel modular group $\Gamma_{2}:=S p_{2}(\mathbb{Z})$ also acts on $\mathbb{S}_{2}$ by fractional linear transformation. Let $\Gamma$ be a subgroup of $\Gamma_{2}$ of finite index. We denote by $M_{k}(\Gamma)$ (resp. $S_{k}(\Gamma)$ ) the space of Siegel modular forms (resp. Siegel cusp forms) of weight $k$ with respect to $\Gamma$. For any $F \in M_{k}\left(\Gamma_{2}(\mathcal{O})\right.$, $\left.\operatorname{det}^{-k / 2}\right)\left(k\right.$ : even), the restriction $\left.F\right|_{\mathbb{S}_{2}}$ becomes a Siegel modular form in $M_{k}\left(\Gamma_{2}\right)$ (cf. Dern-Krieg [3], Corollary 1).

Each $F \in M_{k}\left(\Gamma_{2}\right)$ admits a Fourier expansion of the form

$$
F(Z)=\sum_{0 \leq T \in \Lambda_{2}} A(T ; F) \exp [2 \pi i \operatorname{tr}(T Z)]
$$

where

$$
\Lambda_{2}=\left\{\left(\begin{array}{cc}
a & \frac{c}{2} \\
\frac{c}{2} & b
\end{array}\right) \in M_{2}(\mathbb{Q}) \mid a, b, c \in \mathbb{Z}\right\} .
$$

The Maass space $\mathcal{M}\left(\Gamma_{2}\right)$ consists of Siegel modular forms $F$ characterized by

$$
A(T ; F)=A\left(\left(\begin{array}{cc}
a & \frac{c}{2} \\
\frac{c}{2} & b
\end{array}\right) ; F\right)=\sum_{0<d \mid \varepsilon(T)} d^{k-1} A\left(\left(\begin{array}{cc}
1 & \frac{c}{2 d} \\
\frac{c}{2 d} & \frac{a b}{d^{2}}
\end{array}\right) ; F\right),
$$

where

$$
\varepsilon(T)=\varepsilon\left(\left(\begin{array}{cc}
a & \frac{c}{2} \\
\frac{c}{2} & b
\end{array}\right)\right):=\max \left\{l \in \mathbb{N} \mid l^{-1} T \in \Lambda_{2}\right\}=\operatorname{gcd}(a, b, c) .
$$


An example of Siegel modular form in the Maass space is the Siegel Eisenstein series

$$
E_{k}(Z):=\sum_{\left(\begin{array}{l}
* * \\
C
\end{array}\right):\left\{\left(\begin{array}{l}
* * \\
0 *
\end{array}\right)\right\} \backslash \Gamma_{2}} \operatorname{det}(C Z+D)^{-k} .
$$

If $k \geq 4$ is even, then $E_{k} \in \mathcal{M}_{k}\left(\Gamma_{2}\right)$.

Proposition 2.1. (Dern-Krieg, [3], Theorem 1, Corollary 2). Assume that $\mathbb{K}$ is an imaginary quadratic field of class number 1 . For an even integer $k$ with $k \geq 4$ and the Hermitian Eisenstein series $E_{k, \mathbb{K}}$, we have

$$
\left.E_{k, \mathbb{K}}\right|_{\mathbb{S}_{2}} \in \mathcal{M}_{k}\left(\Gamma_{2}\right) .
$$

\section{Fourier coefficient of Eisenstein Series}

In this section, we present explicit formulas for the Fourier coefficient of Eisenstein series for the Hermitian modular group and the Siegel modular group of degree 2 .

3.1. The Hermitian modular case. In [9, Krieg presented an explicit formula for the Fourier coefficient $A\left(H ; E_{k, \mathbb{K}}\right)$ for the case in which the class number of $\mathbb{K}$ is 1 . To describe this, we introduce Krieg's function $G_{\mathbb{K}}(s, N): \mathbb{N} \times \mathbb{Z} \longrightarrow \mathbb{Q}$, which is defined as

$$
G_{\mathbb{K}}(s, N)= \begin{cases}\frac{1}{1-\chi_{\mathbb{K}}(N)}\left(\sigma_{s, \chi_{\mathbb{K}}}(N)-\sigma_{s, \chi_{\mathbb{K}}}^{*}(N)\right) & \text { if } N>0, \chi_{\mathbb{K}}(N) \neq 1, \\ -\frac{B_{s+1, \chi_{\mathbb{K}}}}{2(s+1)} & \text { if } N=0, \\ 0 & \text { otherwise, }\end{cases}
$$

where $\chi_{\mathbb{K}}$ is the Kronecker character of $\mathbb{K}, \chi_{\mathbb{K}}=\left(\frac{d_{\mathbb{K}}}{*}\right)=\chi_{d_{\mathbb{K}}}$,

$$
\sigma_{s, \chi_{\mathbb{K}}}(N):=\sum_{0<d \mid N} \chi_{\mathbb{K}}(d) d^{s}, \quad \sigma_{s, \chi_{\mathbb{K}}}^{*}(N):=\sum_{0<d \mid N} \chi_{\mathbb{K}}(N / d) d^{s},
$$

and $B_{m, \chi}$ is the generalized Bernoulli number.

Theorem 3.1. (Krieg [9], Dern-Krieg [3]). Assume that the class number of $\mathbb{K}$ is

1. Then, the Fourier coefficient $A\left(H ; E_{k, \mathbb{K}}\right)(k \geq 4)$ is as follows:

(1) If $H>0$, then

$$
A\left(H ; E_{k, \mathbb{K}}\right)=\frac{4 k(k-1)}{B_{k} \cdot B_{k-1, \chi_{\mathbb{K}}}} \sum_{0<d \mid \varepsilon(H)} d^{k-1} G_{\mathbb{K}}\left(k-2, \frac{\left|d_{\mathbb{K}}\right| \operatorname{det}(H)}{d^{2}}\right)
$$

where $\varepsilon(H):=\max \left\{l \in \mathbb{N} \mid l^{-1} H \in \Lambda_{2}(\mathbb{K})\right\}$.

(2) If $\operatorname{rank}(H)=1$, then

$$
A\left(H ; E_{k, \mathbb{K}}\right)=-\frac{2 k}{B_{k}} \sigma_{k-1}(\varepsilon(H)) .
$$

(3) $A\left(0_{2} ; E_{k, \mathbb{K}}\right)=1$. 
3.2. The Siegel modular case. An explicit formula for the Fourier coefficient $A\left(T ; E_{k}\right) \quad\left(E_{k}\right.$ : Siegel Eisenstein series) was first obtained by Kaufhold [7] and Maass [10]. Second, Eichler and Zagier presented an explicit formula obtained by means of Cohen's function $H(r, N)$ (cf. [4).

Cohen [1] introduced a function $H: \mathbb{N} \times \mathbb{Z} \longrightarrow \mathbb{Q}$ given by

$$
H(r, N)=L\left(1-r, \chi_{D}\right) \sum_{0<d \mid f} \mu(d) \chi_{D}(d) d^{r-1} \sigma_{2 r-1}(f / d)
$$

in the case that $r$ and $N$ satisfy $(-1)^{r} N=D f^{2}$, where $D$ is a fundamental discriminant and $f \in \mathbb{N}$. Here, $L(s, \chi)$ is the $L$-function with character $\chi$, and $\mu$ is the Möbius function. Refer to [1] for the precise definition of $H(r, N)$.

Theorem 3.2. (Eichler and Zagier [4]). The Fourier coefficient $A\left(T ; E_{k}\right)$ is given as follows:

(1) If $T>0$, then

$$
A\left(T ; E_{k}\right)=\frac{4 k(k-1)}{B_{k} \cdot B_{2 k-2}} \sum_{0<d \mid \varepsilon(T)} d^{k-1} H\left(k-1, \frac{4 \operatorname{det}(T)}{d^{2}}\right) .
$$

(2) If $\operatorname{rank}(T)=1$, then

$$
A\left(T ; E_{k}\right)=-\frac{2 k}{B_{k}} \sigma_{k-1}(\varepsilon(T)) .
$$

(3) $A\left(0_{2} ; E_{k}\right)=1$.

\section{Construction of the Siegel Cusp form and its application}

In the remainder of the present paper, we assume that

$$
\mathbb{K}=\mathbb{Q}(i): \text { the Gaussian field. }
$$

In this case,

$$
\mathcal{O}=\mathbb{Z}[i]=\{a+b i \mid a, b \in \mathbb{Z}\}, \quad \chi_{\mathbb{K}}=\chi_{-4}=\left(\frac{-4}{*}\right) .
$$

Moreover, the lattice $\Lambda_{2}(\mathbb{K})$ appearing in the Fourier expansion of Hermitian modular forms is given as

$$
\Lambda_{2}(\mathbb{Q}(i))=\left\{\left(\begin{array}{cc}
a & \frac{c+d i}{2} \\
\frac{c-d i}{2} & b
\end{array}\right) \in \operatorname{Her}_{2}(\mathbb{Q}(i)) \mid a, b, c, d \in \mathbb{Z}\right\} .
$$

4.1. Restriction of the Hermitian Eisenstein series. As mentioned in Section 2 , the restriction of $F \in M_{k}\left(\Gamma_{2}(\mathbb{Z}[i])\right.$, det $\left.^{k / 2}\right)$ to the Siegel half-space $\mathbb{S}_{2}$ becomes a Siegel modular form, namely,

$$
\left.F\right|_{\mathbb{S}_{2}} \in M_{k}\left(\Gamma_{2}\right) .
$$

Moreover, the relation between $A\left(T ;\left.F\right|_{\mathbb{S}_{2}}\right)$ and $A(H ; F)$ is

$$
A\left(T ;\left.F\right|_{\mathbb{S}_{2}}\right)=A\left(\left(\begin{array}{cc}
a & \frac{c}{2} \\
\frac{c}{2} & b
\end{array}\right) ;\left.F\right|_{\mathbb{S}_{2}}\right)=\sum_{\substack{s \in \mathbb{Z} \\
s^{2} \leq 4 a b-c^{2}=4 \operatorname{det}(T)}} A\left(\left(\begin{array}{cc}
a & \frac{c+s i}{2} \\
\frac{c-s i}{2} & b
\end{array}\right) ; F\right) .
$$

For even $k \geq 4$, we consider the difference between Siegel Eisenstein series $E_{k}$ and the restriction of Hermitian Eisenstein series $E_{k, \mathbb{Q}(i)}$ :

$$
f_{k}:=E_{k}-E_{k, \mathbb{Q}(i)} \mid \mathbb{S}_{2}
$$


Lemma 4.1. The Siegel modular form $f_{k}$ defined above is a cusp form in the Maass space $\mathcal{M}_{k}\left(\Gamma_{2}\right)$ :

$$
f_{k} \in \mathcal{M}_{k}\left(\Gamma_{2}\right) \cap S_{k}\left(\Gamma_{2}\right) .
$$

Proof. By Proposition 2.1 we have $f_{k} \in \mathcal{M}_{k}\left(\Gamma_{2}\right)$. (Note that the class number of $\mathbb{Q}(i)$ is 1.) It is known that the Maass space $\mathcal{M}_{k}\left(\Gamma_{2}\right)$ contains only Eisenstein series and cusp forms (cf. [4]). Therefore, it is enough to consider the zero Fourier coefficient $A\left(0_{2} ; f_{k}\right)$. Since $A\left(0_{2} ; E_{k}\right)=A\left(0_{2} ; E_{k, \mathbb{Q}(i)} \mid \mathbb{S}_{2}\right)=1$, we have $A\left(0_{2}, f_{k}\right)=$ 0 . This shows that $f_{k} \in S_{k}\left(\Gamma_{2}\right)$.

Theorem 4.2. Let $k$ be an even integer such that $k \geq 4$. Then there exists a Siegel cusp form $F_{k}$ contained in the Maass space $\mathcal{M}_{k}\left(\Gamma_{2}\right)$ such that the Fourier coefficient $A\left(T ; F_{k}\right)$ is given as

$$
\begin{aligned}
& A\left(T ; F_{k}\right)=\sum_{0<d \mid \varepsilon(T)} d^{k-1} \alpha_{k}\left(4 \operatorname{det}(T) / d^{2}\right), \\
& \alpha_{k}(N)=H(k-1, N)-\frac{B_{2 k-2}}{B_{k-1, \chi-4}} \sum_{\substack{s \in \mathbb{Z} \\
s^{2} \leq N}} G_{\mathbb{Q}(i)}\left(k-2, N-s^{2}\right) .
\end{aligned}
$$

Proof. If we normalize $f_{k}$ as

$$
F_{k}:=\frac{B_{k} \cdot B_{2 k-2}}{4 k(k-1)} f_{k},
$$

then $F_{k} \in \mathcal{M}_{k}\left(\Gamma_{2}\right) \cap S_{k}\left(\Gamma_{2}\right)$ satisfies the required properties.

Remark 4.3. As described later, the Siegel cusp form $F_{k}$ vanishes identically for small $k$ (see the proof of Theorem [5.2). We shall refer to the non-vanishing property of $F_{k}$ here. We fix $T=\left(\begin{array}{cc}1 & 1 / 2 \\ 1 / 2 & 1\end{array}\right) \in \Lambda_{2}$. Then, by Theorem 4.2, the corresponding Fourier coefficient is given by

$$
A\left(T ; F_{k}\right)=-\frac{B_{k-1, \chi_{-3}}}{k-1}+\frac{B_{2 k-2}}{B_{k-1, \chi_{-4}}}\left\{\left(3^{k-2}-1\right)+2\left(2^{k-2}-1\right)\right\} .
$$

We consider the asymptotic approximation of the right side as $k \rightarrow \infty$. In general, we have

$$
\left|B_{2 m}\right| \sim 4 \sqrt{\pi m}\left(\frac{m}{\pi e}\right)^{2 m} \quad(m \rightarrow \infty)
$$

and

$$
\left|B_{2 m-1, \chi}\right| \sim \frac{2}{\sqrt{f}}\left(\frac{f}{2 \pi}\right)^{2 m-1}(2 m-1) ! \quad(m \rightarrow \infty),
$$

where $f=f_{\chi}$ is the conductor of $\chi$ (cf. [12, Exercises 4.3(b)). From these approximations, we see that the Fourier coefficient $A\left(T ; F_{k}\right)$ does not vanish for sufficiently large $k$. 


\section{Applications}

5.1. Cohen's function and Krieg's function. As an application of Theorem 4.2 , we present a new description of Cohen's function.

Proposition 5.1. (Cohen [1], Proposition 4.2). Set

$$
\begin{gathered}
s_{2}(n):=\sum_{d \mid n}\left((n / d)^{2}-2 d^{2}\right)\left(\frac{-4}{d}\right) \text { for } n>0, \\
s_{2}(0)=1 / 2, \\
s_{4}(n)=\sum_{d \mid n}\left((n / d)^{4}-2 d^{4}\right)\left(\frac{-4}{d}\right)+(3 / 2) \sum_{n=x^{2}+y^{2}}\left(x^{4}-6 x^{2} y^{2}+y^{4}\right) \text { for } n>0, \\
s_{4}(0)=-5 / 2 .
\end{gathered}
$$

Then

$$
\begin{aligned}
& H(3, N)=-\frac{1}{126} \sum_{\substack{s \in \mathbb{Z} \\
s^{2} \leq N}} s_{2}\left(N-s^{2}\right), \\
& H(5, N)=\frac{1}{330} \sum_{\substack{s \in \mathbb{Z} \\
s^{2} \leq N}} s_{4}\left(N-s^{2}\right) .
\end{aligned}
$$

Using Theorem 4.2, we present a new description of $H(r, N)$.

Theorem 5.2. Let $G_{\mathbb{Q}(i)}(s, N)$ be Krieg's function for $\mathbb{K}=\mathbb{Q}(i)$. Then,

$$
\begin{aligned}
& H(3, N)=\frac{1}{63} \sum_{\substack{s \in \mathbb{Z} \\
s^{2} \leq N}} G_{\mathbb{Q}(i)}\left(2, N-s^{2}\right), \\
& H(5, N)=-\frac{1}{165} \sum_{\substack{s \in \mathbb{Z} \\
s^{2} \leq N}} G_{\mathbb{Q}(i)}\left(4, N-s^{2}\right), \\
& H(7, N)=\frac{1}{183} \sum_{\substack{s \in \mathbb{Z} \\
s^{2} \leq N}} G_{\mathbb{Q}(i)}\left(6, N-s^{2}\right) .
\end{aligned}
$$

Proof. The fact that $S_{k}\left(\Gamma_{2}\right)=\{0\}$ for $k=4,6,8$ implies that $F_{k} \equiv 0$ if $k=4,6,8$, where $F_{k}$ is the Siegel cusp form given in Theorem 4.2. Therefore, we obtain

$$
H(k-1, N)=\frac{B_{2 k-2}}{B_{k-1, \chi-4}} \sum_{\substack{s \in \mathbb{Z} \\ s^{2} \leq N}} G_{\mathbb{Q}(i)}\left(k-2, N-s^{2}\right)
$$

for $k=4,6,8$. Thus, we obtain the required formulas.

5.2. Igusa's cusp forms. In [5], Igusa studied the structure of the graded ring of Siegel modular forms of degree 2.

Theorem 5.3 (Igusa [5]). There exist two Siegel cusp forms $X_{10}$ and $X_{12}$ of weights 10 and 12 , respectively, and the graded ring $\bigoplus_{k \in 2 \mathbb{Z}} M_{k}\left(\Gamma_{2}\right)$ is generated by four Siegel modular forms $E_{4}, E_{6}, X_{10}$, and $X_{12}$, where $E_{k}$ is the Siegel Eisenstein series:

$$
\bigoplus_{k \in 2 \mathbb{Z}} M_{k}\left(\Gamma_{2}\right)=\mathbb{C}\left[E_{4}, E_{6}, X_{10}, X_{12}\right] \text { (polynomial ring). }
$$


If $k=10,12$, then the Siegel cusp form of weight $k$ is uniquely determined up to constant. Then, $X_{10}$ and $X_{12}$ are normalized as

$$
A\left(\left(\begin{array}{cc}
1 & \frac{1}{2} \\
\frac{1}{2} & 1
\end{array}\right) ; X_{10}\right)=A\left(\left(\begin{array}{cc}
1 & \frac{1}{2} \\
\frac{1}{2} & 1
\end{array}\right) ; X_{12}\right)=1 .
$$

Then, all of the Fourier coefficients of $X_{k}(k=10,12)$ are rational integers (cf. Igusa [6]).

Theorem 5.4. Let $f_{k}$ be the Siegel cusp form defined in (*). Then,

$$
f_{k}=E_{k}-\left.E_{k, \mathbb{Q}(i)}\right|_{\mathbb{S}_{2}}=c_{k} \cdot X_{k} \quad(k=10,12),
$$

where

$$
c_{10}=-\frac{2^{14} \cdot 3^{4} \cdot 5^{2} \cdot 7 \cdot 11}{277 \cdot 43867}, \quad c_{12}=-\frac{2^{14} \cdot 3^{6} \cdot 5^{6} \cdot 7^{2} \cdot 13}{19 \cdot 131 \cdot 593 \cdot 691 \cdot 2659} .
$$

Proof. Since $S_{k}\left(\Gamma_{2}\right)=\mathbb{C} \cdot X_{k}$ for $k=10,12$ (Theorem [5.3), $f_{k}=c_{k} \cdot X_{k}$ for some constant $c_{k}$. The explicit values of $c_{k}$ are obtained by direct calculation of the Fourier coefficients of both sides.

Corollary 5.5. For a primitive $T \in \Lambda_{2}$ (i.e., $\left.\varepsilon(T)=1\right)$, we have

$$
\begin{aligned}
& A\left(T ; X_{10}\right)=-\frac{5263}{12800} H(9,4 \operatorname{det}(T))-\frac{43867}{12096000} \sum_{\substack{s \in \mathbb{Z} \\
s^{2} \leq 4 \operatorname{det}(T)}} G_{\mathbb{Q}(i)}\left(8,4 \operatorname{det}(T)-s^{2}\right) \\
& A\left(T ; X_{12}\right)=\frac{1161983}{151200000} H(11,4 \operatorname{det}(T))-\frac{77683}{453600000} \sum_{\substack{s \in \mathbb{Z} \\
s^{2} \leq 4 \operatorname{det}(T)}} G_{\mathbb{Q}(i)}\left(10,4 \operatorname{det}(T)-s^{2}\right) .
\end{aligned}
$$

Proof. By Theorems 4.2 5.4, we have

$$
\begin{aligned}
& A\left(T ; X_{k}\right)=c_{k}^{-1} \cdot \frac{4 k(k-1)}{B_{k} \cdot B_{2 k-2}} \alpha_{k}(4 \operatorname{det}(T)) \\
& =\frac{4 k(k-1)}{c_{k} \cdot B_{k} \cdot B_{2 k-2}}\left\{H(k-1,4 \operatorname{det}(T))-\frac{B_{2 k-2}}{B_{k-1, \chi_{-4}}} \sum_{\substack{s \in \mathbb{Z} \\
s^{2} \leq 4 \operatorname{det}(T)}} G_{\mathbb{Q}(i)}\left(k-2,4 \operatorname{det}(T)-s^{2}\right)\right\} .
\end{aligned}
$$

Remark 5.6. -adic Siegel cusp forms: Combining the results from [8] and [11] we immediately see that for a prime $p \equiv 3(\bmod 4)$ and $k_{m}:=2+(p-1) p^{m}$ the sequence $f_{k_{m}}$ converges $p$-adically to a true modular form $\widetilde{f}$ for $\Gamma_{0}(p)$. Based on some numerical evidence we conjecture that $\widetilde{f}$ is a cusp form.

\section{ACKNOWLEDGEMENT}

The authors thank the referee for many valuable comments.

\section{REFERENCES}

1. H. Cohen, Sums involving the values at negative integers of L-functions of quadratic characters. Math. Ann. 217 (1975), 271-285. MR0382192 (52:3080)

2. T. Dern, Hermitesche Modulformen zweiten Grades. Verlag Mainz, Aachen, 2001.

3. T. Dern, A. Krieg, Graded rings of Hermitian modular forms of degree 2, Manuscripta Math. 110 (2003), 251-256. MR.1962537 (2004b:11059) 
4. M. Eichler, D. Zagier, The Theory of Jacobi Forms. Birkhäuser, Boston, Basel, Stuttgart, 1985. MR781735 (86j:11043)

5. J.-I. Igusa, Siegel modular forms of genus two. Amer. J. Math. 84 (1962), 175-200. MR0141643(25:5040)

6. J.-I. Igusa, On the ring of modular forms of degree two over $\mathbb{Z}$. Amer. J. Math. 101 (1979), 149-183. MR527830(80d:10039)

7. G. Kaufhold, Dirichletsche Reihe mit Funktionalgleichung in der Theorie der Modulfunktion 2. Grades. Math. Ann. 137 (1959), 454-476. MR0121485 (22:12223)

8. T. Kikuta, S. Nagaoka, On a correspondence between p-adic Siegel-Eisenstein series and genus theta series. Acta Arithmetica 134 (2008), 111-126. MR2429640 (2009e:11083)

9. A. Krieg, The Maass space on the Hermitian half-space of degree 2. Math. Ann. 289 (1991), 663-681. MR1103042 (93d:11051)

10. H. Maass, Die Fourierkoeffizienten der Eisensteinreihen zweiten Grades. Dan. Vid. Selsk. 34 (1964), 1-25. MR0171758 (30:1985)

11. S. Nagaoka, On p-adic Hermitian Eisenstein series. Proc. Amer. Math. Soc. 134 (2006), 2533-2540. MR2213730 (2007h:11054)

12. L. C. Washington, Introduction to Cyclotomic Fields. Springer Verlag, New York, Heidelberg, Berlin, 1982. MR718674 (85g:11001)

13. H. Yoshida, Siegel's modular forms and the arithmetic of quadratic forms. Invent. Math. 60 (1980), 193-248. MR586427 (81m:10051)

Department of Mathematics, Kinki University, Higashi-Osaka, Osaka 577-8502, Japan

E-mail address: nagaoka@math.kindai.ac.jp

Department of Mathematics, Kinki University, Higashi-Osaka, Osaka 577-8502, Japan

E-mail address: yoshi-nakamura@math.kindai.ac.jp 